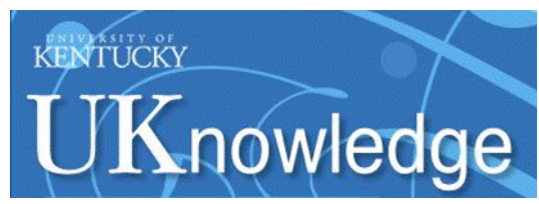

University of Kentucky

UKnowledge

\title{
Systematic Review of Ambulatory Transitional Care Management (TCM) Visits on Hospital 30-Day Readmission Rates
}

\author{
Karen L. Roper \\ University of Kentucky, karen.roper@uky.edu \\ Jonathan Ballard \\ University of Kentucky, jonathan.ballard@uky.edu \\ Wade Rankin \\ University of Kentucky, wademrankin@uky.edu \\ Roberto Cardarelli \\ University of Kentucky, roberto.cardarelli@uky.edu
}

Follow this and additional works at: https://uknowledge.uky.edu/familymedicine_facpub

Part of the Medicine and Health Sciences Commons

Right click to open a feedback form in a new tab to let us know how this document benefits you.

\section{Repository Citation}

Roper, Karen L.; Ballard, Jonathan; Rankin, Wade; and Cardarelli, Roberto, "Systematic Review of Ambulatory Transitional Care Management (TCM) Visits on Hospital 30-Day Readmission Rates" (2015). Family and Community Medicine Faculty Publications. 3.

https://uknowledge.uky.edu/familymedicine_facpub/3

This Article is brought to you for free and open access by the Family and Community Medicine at UKnowledge. It has been accepted for inclusion in Family and Community Medicine Faculty Publications by an authorized administrator of UKnowledge. For more information, please contact UKnowledge@lsv.uky.edu. 


\section{Systematic Review of Ambulatory Transitional Care Management (TCM) Visits on Hospital 30-Day Readmission Rates}

Digital Object Identifier (DOI)

https://doi.org/10.1177/1062860615615426

Notes/Citation Information

Published in American Journal of Medical Quality, v. 32, issue 1, p. 19-26.

The copyright holder has granted the permission for posting the article here.

The document available for download is the authors' post-peer-review final draft of the article. The figure, tables, and appendix are available as the additional files listed at the end.

This article is available at UKnowledge: https://uknowledge.uky.edu/familymedicine_facpub/3 
Systematic review of ambulatory transitional care management (TCM) visits on hospital 30-day readmission rates

Karen L. Roper, PhD Jonathan Ballard, MD, MPH, MPhil Wade Rankin, DO Roberto Cardarelli, DO, MPH

Affiliation (all authors):

University of Kentucky, Department of Family \& Community Medicine / Division of Community Medicine, Lexington, $\mathrm{KY}$

Correspondence:

Karen L. Roper; University of Kentucky College of Medicine, Department of Family \& Community Medicine / Division of Community Medicine, 2195 Harrodsburg Road, Suite 125 Lexington, Kentucky 40504 -3504, USA. Email: karen.roper@uky.edu. Phone: (859) 257-4880. Fax: (859) 323-6661

Word Count: $[3,292]$

Tables: 3

Figures: 1

Appendix: 1

Running title: Systematic Review of TCM 


\section{Funding Acknowledgements:}

This research received no specific grant from any funding agency in the public, commercial, or not-for-profit sectors. 


\section{Abstract}

Purpose:

To reduce readmission rates and prevent adverse outcomes after discharge, hospitals have begun implementing "transitional care" initiatives. This systematic review identifies research on the particular set of services now reimbursable by Medicare ("transitional care management", TCM) and evaluates the studies for program effectiveness.

Methods:

Results of three databases were screened for peer reviewed journal articles published between January 2004-2015 that report on readmissions of adults in the US healthcare system under the Medicare TCM bundle. ClinicalTrials.gov was queried for funded studies.

Results:

Of 969 identified studies, 77 met inclusion criteria for relevance to transitional care and appropriateness of population and setting. Of these, only three articles incorporated all required elements for TCM service. Although two were program improvement designs and none were randomized control studies, each report reduced readmission rates.

Conclusions:

Evidence for TCM effectiveness is limited. Additional study of TCM implementation and programmatic support for TCM is warranted.

Keywords: Transitional care; Systematic review, 30-day readmission, chronic disease 


\section{INTRODUCTION}

\section{Background}

From 2007 to 2011 , 19\% of patients were readmitted within 30 days of a hospital discharge, ${ }^{1}$ with costs estimated at $\$ 15$ billion. $^{2}$ After tying Medicare reimbursement of hospitals to their readmission rate under a provision of Section 3025 of the Affordable Care Act (ACA), claims data are being closely monitored for a reduction in these rates, and hospitals are working on multiple fronts to address the issues that lead to patient rehospitalizations. ${ }^{3}$ These efforts are especially challenging since many of these patients have multiple co-morbidities that result in complications and readmissions that are unrelated to the original admitting condition, ${ }^{4,5}$ and therefore are not well addressed by a disease-specific care model. ${ }^{6,7}$ Indeed, almost a third of the US population is now living with two or more of the nation's most deadly chronic conditions, such as diabetes, hypertension, heart disease, or cancer. ${ }^{8}$ These patients have highcomplexity medical decision-making and medication needs, and incur costly acute events and hospitalizations $^{9}$ that are exacerbated by a compromised quality of life ${ }^{10}$ and, quite often, depression. ${ }^{11}$

The Agency for Healthcare Research and Quality (AHRQ) has led the call for progress in addressing modifiable behavioral factors that could affect outcomes and specifically, readmissions. The AHRQ identifies conditions such as chronic pain and cardiovascular disease as ones that are predominantly met in the primary care setting and for which significant treatment limitations occur in the area of care integration and within vulnerable populations. ${ }^{12}$ Establishing a continuum of care that will address the needs of these patients and the ensuing problem of readmissions requires solutions that reach across and encompass the entire health 
system to identified areas of disconnect. ${ }^{13-15}$ For example, Medicare payment patterns reveal a failure by over $50 \%$ of readmitted patients to visit a physician's office between the time of discharge and rehospitalization. ${ }^{16}$ This is especially concerning for older adults, as research shows they are twice as likely to incur post-discharge problems when their primary care providers were unaware of their hospitalization. ${ }^{17}$ Care transitions, or handoffs, among care providers present additional system-associated challenges including high rates of medication reconciliation error, ${ }^{18,19}$ and problems inherent in the transfer of medical information and/or in the patient and caregiver's knowledge and skills in handling medical information. ${ }^{20}$ Failure to implement a successful model to address these modifiable risk factors and prevent patient readmission also carries a penalty to the hospital, since readmission penalties were inacted in fiscal year 2013 and now can total as much as 3\% of inpatient claims, based on adjusted readmission rates. ${ }^{21}$

\section{Transitional Care Management}

Initiatives termed "transitional care interventions" have been developed to provide what Naylor, et al. defined as "a broad range of time-limited services" aimed at "the safe and timely transfer of patients from one level of care to another or from one type of setting to another" (p. 747). ${ }^{22}$ In an initial 2011 review, the authors point to the diversity of transitional care interventions that had been undertaken, while noting the lack of sufficient program description that would be necessary to establish the relative efficacy of the individual interventions. ${ }^{3}$ Similar limitations were still noted in more recent systematic reviews, ${ }^{23-25}$ and thus no clear set of intervention components had been established to define a successful transitional care 
intervention. Moreover, while the Institute of Medicine (IOM) has called for studies that compare the effectiveness of comprehensive care coordination programs with usual care in managing patients with chronic disease, ${ }^{26}$ previous reviews have noted that observational designs are currently the predominant basis of evidence. ${ }^{23}$

Beginning in 2013, Medicare reimbursement for specific ambulatory (outpatient) clinic services (including: post-discharge communication, follow-up scheduling, education, and adherence assessment) known as transitional care management (TCM) was initiated as a means of facilitating services for patients after being discharged from a hospital, or another eligible facility, with high-complexity medical decision making needs. Table 1 and associated references ${ }^{27,28}$ describe the specific current procedural terminology (CPT) coding requirements that were adopted in 2013. These outpatient and billable TCM visits are the focus of this review, as other hospital-initiated interventions, such as the E. Coleman's Care Transitions Intervention ${ }^{20,29}$ and the National Society of Hospital Medicine's BOOST (Better Outcomes for Older adults through Safe Transitions) model $^{30}$ are already well described in the literature. Also, as these models are hospital-initiated, they would not apply to other, outpatient or clinic settings where these TCM billing codes are most likely to be used, and given provisions that the codes be used by a single health care professional once per beneficiary during the TCM period. This review was conducted to assess the status of research that utilize TCM as a distinct intervention in the ambulatory setting, summarize the findings and evaluate the evidence for establishing TCM effectiveness, and offer strategies to help improve this evidence base.

\section{METHODS}




\section{Search Strategy and Article Selection}

This systematic review used the IOM's standards for systematic reviews ${ }^{31}$ to evaluate the evidence for transitional care interventions provided as an outpatient service for adults in the US healthcare system. We searched MEDLINE, the Cochrane Library (The Cochrane Central Register of Controlled Trials Database), and the CINAHL Database from January 1, 2004 to January 1, 2015 for peer-reviewed publications. We searched ClinicalTrials.gov for ongoing and completed trials related to the key questions. We manually searched reference lists from relevant reviews and eligible studies, and evaluated the citation index of background articles using MEDLINE for any relevant citations that our searches may have missed. With the assistance of a professional medical librarian, we used relevant Medical Subject Headings (MeSH) terms and text words including and related to TCM and to patient discharge or readmission (search strategies shown in Table 2).

Other than the exclusion of non-English articles, editorials, letters and/or practice guidelines, and review articles, we did not further restrict our search with additional limits, preferring instead to individually evaluate abstracts according to inclusion and exclusion criteria guided by the PICOTS (populations, interventions, comparators, outcomes, timing, settings) framework. ${ }^{32}$ The PICOTS typology is considered a "vital part" of systematic reviews according to methods guidelines published by the AHRQ. ${ }^{33}$ PICOTS makes the context for evaluating the applicability of a review clear and explicit, by defining the limits of the literature search selections. As applied here, these limits include: Population = Adults (ages 18 years or older). Interventions $=$ transitional care interventions. All interventions aimed at reducing readmissions were included in the first screening set. Studies were excluded at the second stage of review if 
they failed to follow all aspects of the required procedures to qualify under the CPT-coded criterion for a TCM visit. Comparators = Usual care, routine care, or standard care. Outcomes = Readmission rates. Other outcomes (e.g., mortality, quality of life, functional status change) were included at the first stage of review. Timing $=$ Readmission within 30 days. Time Period $=$ Last 10 years (studies published after 2004). Setting $=$ Hospital Interventions occurring before discharge or initiated as an outpatient following the index hospitalization or during the transition from inpatient to outpatient care. Excluded were all other settings; specifically, TCM within a skilled nursing facility, rehabilitation center, psychiatric facility or surgical setting. Except for the surgical discharge, each of these exclusionary settings is accepted for reimbursement for TCM services; however we purposed to limit our search to TCM in the ambulatory setting. These excluded settings were perceived by our team as less likely to result in a home discharge, and/or result in timely follow-up with a primary care team.

\section{RESULTS}

\section{Search Results}

Search strategy results with reasons for exclusion are listed in Figure 1. The search initially yielded 1,031 records, of which 969 were of the appropriate record type. Excluded were articles that were: commentary $(n=21)$, a review article $(n=9)$, or a protocol or program description, survey of patient/staff experience of a care program, or cost-analytics description $(n=22)$. The abstracts from another 10 identified articles were not yet available or inaccessible, and could not be properly evaluated. Of the qualifying articles, 892 were further excluded at this stage of the review, primarily because the intervention would not qualify as transitional care $(n=800)$. 
The majority of the articles excluded at this stage concerned the issues and difficulties that underlie high levels of readmission; articles of this type described patterns or predictors (e.g. patient characteristics) of emergency department (ED) use, or describe patient needs and discharge experiences but did not examine a particular care model. Others described care services that were home-based or not furnished from one of the discharge settings covered by CMS (e.g., emergency department or trauma center, substance treatment centers, prisons). The remaining 77 articles met inclusion criteria for appropriateness of the population and setting, and all contained relevant aspects of a transitional care intervention in stage 1 of the review process (see Appendix for citations: Excluded Transitional Care Literature).

In a second phase of review, articles were excluded if the intervention was insufficient to qualify as TCM under the 2013 CPT coding requirements. Articles and trial descriptions were excluded at this stage if they did not offer all components to meet billing standards for TCM methodology (Table 1), including studies that may have included one or more of the requirements for transitional care (phone call or other interactive initial contact, medication reconciliation, and/or a face-to-face follow-up visit within the allotted time frame), but either did not offer all components to meet billing standards for TCM methodology, or did not make a sufficiently clear description of the TCM process to make that determination. The remaining 3 articles $^{34-36}$ were carefully vetted in the final stage of review.

\section{Evidence for TCM Effectiveness}

The three identified studies (Table 3) that utilized a fully reimbursable TCM approach each reported reduced all-cause hospital re-admissions within the first month following discharge. 
These effects varied from modest (a $1.8 \%$ reduction), ${ }^{34}$ to substantial (approximately $20 \%$ reduction). ${ }^{35,36}$ It is important to qualify these findings, however, since two (Jackson et al., and Tuso et al. $)^{34,36}$ of the three identified studies were institutional improvement designs, and none were randomized trials. The smallest reduction was reported by Tuso et al. ${ }^{34}$ based on implementation of TCM in a large hospital system. Importantly, this was a retrospective analysis of care components that were being implemented throughout the study period rather than a before-after design; in fact, they selected this model for broader implementation based on a much greater (10\%) reduction observed in one of their affiliated hospitals prior to the system roll out. While also a quality improvement design, the study by Jackson et al. ${ }^{36}$ was significant in that it drew from a state-wide patient population across 120 hospitals rather than a regional pool and concentrates on 12-month rather than the first 30-day readmission rates. It also offers a cross-sectional comparison to patients that a nurse manager had not selected for TCM based on risk. To account for potential confounds that could result from this assignment method, the approximately $20 \%$ difference reported between TCM and no-TCM patient readmission rates was calculated only after controlling for substantial patient and hospital characteristics. While we feel this was a reasonable approach, we sought to make a more direct comparison across our three identified studies in Table 3, and so calculated their rate of change in 30-day readmissions without this correction (using change scores across 8 patient risk groups at the first 30-day assessment, the only 30-day rate the authors present). This interpretation of their data would indicate a difference closer to $9 \%$. It is also notable that a small percentage of the lowest risk patients in both the Jackson et al. and Tuso et al. studies received what Jackson et al. characterized as "low intensity" services, and not the full suite of TCM services that would 
have qualified their study to be included in this review. The authors did not report on differences between patients receiving the lower "intensity" of their services; however this would only have reduced the overall magnitude of the effect of TCM in both of these studies.

The one (White et al.) ${ }^{35}$ investigation that was designed to explicitly compare practice groups that did and did not offer TCM reported readmissions from two study groups, which unfortunately were not randomly assigned: Baseline readmissions were $27 \%$ and $26.1 \%$ in control and intervention groups, respectively. The 30-day readmission rate was then assessed each month throughout a 12-month period. The final rate was maintained in the control group (25.9\%), but significantly improved under the conditions of TCM (7.1\%).

As reported by one of the identified studies (Jackson et al.), ${ }^{36}$ the effectiveness of the TCM intervention is likely to vary by degree of patient risk. Using a "clinical risk group" classification of disease burden and severity (scale developed by $3 \mathrm{M}),{ }^{37}$ readmission rates within the first 30 days for the highest risk patients showed the greatest amount of change ( $16 \%$ as compared to $4 \%$ for low risk patients). The interventional program described by Tuso ${ }^{34}$ also incorporated risk for readmission in its determination of which TCM components patients are offered, using LACE scores (a validated measure incorporating length of stay, acute admission through the ED, co-morbidities, and emergency department visits in the past six months). ${ }^{38}$ Unfortunately, this report concentrated on the programmatic features of their intervention, and data relevant to the utility of the LACE score in predicting benefit from TCM was limited to the single site that served as the model for their system-wide TCM roll-out. Based on retrospective analysis of patients admitted at this site, the authors conclude that the 
observed-to-expected number of cases for the highest risk patients identified by LACE was "much greater" than 1.0 (and was "much lower" than 1.0 in the lowest risk patients).

Finally, while each of the three studies of TCM did well to involve multiple institutions in their assessment, two were a study of large hospital systems, and the third study by White et al. was a smaller quasi-experimental study that featured TCM only in university-based practice groups (inpatients from unaffiliated county and community clinics served as non-randomized control practices). Thus, there may be limited generalizability of these three studies to other patient populations (e.g., a small or medium-sized practice clinic).

\section{DISCUSSION}

\section{Quality of Evidence}

Despite several prior reviews and our identification of over 75 identified articles that investigate readmission and other outcomes of both individual and bundled components of transitional care, our analysis of the literature identified only three articles that utilized all of the required elements for TCM service for today's Medicare billing code. Of these studies, two were quality improvement designs that did not feature adequate comparison conditions. The one study that did offer comparison to a no-TCM control (White et al.) ${ }^{35}$ reports the greatest overall difference in 30-day readmission under TCM conditions, but is more limited than the other studies in that TCM was investigated only in the university practice group setting. This study may also be limited in its application to the real-world uptake of TCM, since unlike most prior studies of other transitional care elements, the White et al. analysis did not assess a high-risk elderly 
population or use a risk-stratification measure to identify patients to whom providers might focus their resources.

Nevertheless, each of the three identified studies offer promise that substantial reductions in readmission can still be found even under conditions where readmission rates are not as high under baseline conditions. In the White et al. study, for example, the authors report a $19.9 \%$ reduction in 30 -day readmissions from an original rate of $27 \%$, which compares favorably with other published research on care transitions in which a higher baseline rate of all-cause readmission (e.g., over $35 \%)^{39,40}$ is reported in generally elderly populations. The baseline rates in the Jackson et al. and Tuso et al. studies are even lower $(11.13 \%$ and $12.8 \%$, respectively). Naylor et. al.'s data on elderly heart failure patients, for example, reported a readmission rate over a 12 month follow-up of $55.4 \%$ in the control, as compared $36.4 \%$ in the intervention group. ${ }^{41}$ The lower rates in the studies reviewed here may be attributed to differences in the enrollment criteria: Unlike the Naylor study, none exclusively assessed a highrisk elderly population. The mean age in White et al.'s TCM patients was 43.8; the Jackson et al. study included only Medicaid-enrolled patients, and was limited to adults under age 65 .

\section{Implications and Conclusions}

Despite nationwide advances in curbing readmission rates through quality improvement programs and effective interventions that incorporate aspects of transitional care management, gaps remain in knowledge about the effectiveness of the specific set of services that qualify under CPT codes as TCM. Whereas most care transition programs are hospital-based efforts to ensure appropriate linkage to care, TCM ensures that certain post-discharge activities actually occur. No prior review has systematically selected and summarized studies specific to this 
billing model, and our review was able to identify and summarize the findings from only three studies that currently meet this criterion. Each of these studies offers evidence to suggest that TCM is an effective suite of services, and is able to demonstrate this even when rates of readmissions are already low. However, based on our comments on the quality of this evidence, we must emphasize that a far broader assessment of TCM outcome effectiveness is warranted and especially new research that could meet the IOM's call to utilize a comparative analysis against programs without TCM practice redesign. The limited scope of these studies makes our review particularly relevant to providers who seek to implement and properly evaluate this quality improvement initiative within their own practice setting.

Our search makes clear that this already understudied TCM approach has not been evaluated using the population, approach, setting, and timing that would make the reported results meaningful to many of the individual providers that would most benefit from a billable TCM strategy. Hospital systems and academic medical centers usually have internal capacity and other varying motivators for performing quality improvement strategies, such as TCM. While it was not the focus of this review, others have commented on the failure of prior studies to describe the institutional context of the health system that promoted a successful transitional care strategy. ${ }^{23}$ The effectiveness of TCM across different target settings should be the focus of future research, and should address the scope of challenges and processes that are involved in implementation of TCM processes.

The true value of care transition initiatives is becoming well established in terms of enhanced patient satisfaction and patient equity. ${ }^{42}$ Activation of the patient is at the very center of this work, and other evidence of care management effectiveness is beginning to be 
shown in improved patient self-management behaviors (e.g., dietary and medication adherence) ${ }^{25}$ It is a limitation of this review that we may have missed TCM interventions that may have addressed other measures of quality improvement rather than rate of readmission. Indeed, improvements in quality of life and functional status achieved by the various studied components of transitional care have not yet shown clear clinical significance, ${ }^{25}$ and this will be an important aim in future evaluation of the effects of an outpatient-clinic TCM process in improving patient-centered outcomes. This work should also incorporate further demographic and clinical evaluation of the patient, especially as risk assessment promises to add to our understanding of the highest impact groups for TCM service. Also, despite significant effort from prior literature review, it has not been established which of the components of TCM billing may best reduce hospital readmission, either on their own or in sum. ${ }^{43}$ However, we maintain that the only way to fully assess TCM effectiveness is to use the approach of looking at only studies that implemented all components, as performed in this review. Single or other bundled components might then be compared against the full suite of services.

The many changes in ACA reform have created a "clash" between medical practice and health policy ${ }^{44}$ that has resulted in providers feeling overwhelmed and less open to "new" change. ${ }^{45}$ A potential barrier is simply the lack of desire to implement a new clinical process. The decision to support TCM services in a model of care certainly embraces a renewed focus on patient-centered care, and reimbursement by CMS and other insurers should increase the likelihood that clinics will sustain TCM programs. In addition to the call for further study of TCM effectiveness, it is imperative that appropriate resources be allocated to assist clinics as they implement a TCM program. Development of an accessible "how-to" guide or practice-based 
toolkit should be an important priority if the TCM model is to meet its intended goal of reducing avoidable hospital readmissions, and addressing the greater needs of the chronically ill. 
Systematic Review of TCM / 17

\section{Acknowledgements:}

The authors are grateful for the help of Mark Ingram, Clinical reference librarian, for his guidance in developing the search strategies and terms that formed the basis for this review.

Declaration of Conflict of Interest:

None. 


\section{References}

1. Gerhardt G, Yemane A, Hickman P, Oelschlaeger A, Rollins E, Brennan N. Medicare readmission rates showed meaningful decline in 2012. Medicare Medicaid Res Rev. 2013;3(2).

2. The Medicare Payment Advisory Commission. Report to the Congress: Promoting Greater Efficiency in Medicare. Ch. 5. Payment policy for inpatient readmissions. Washington, DC: MedPAC; June, 2007.

3. Hansen LO, Young RS, Hinami K, Leung A, Williams MV. Interventions to reduce 30-day rehospitalization: a systematic review. Arch Int Med. Oct 18 2011;155(8):520-528.

4. Sacks GD, Dawes AJ, Russell MM, et al. Evaluation of hospital readmissions in surgical patients: do administrative data tell the real story? JAMA Surgery. Aug 2014;149(8):759-764.

5. Dharmarajan K, Hsieh AF, Lin Z, et al. Diagnoses and timing of 30-day readmissions after hospitalization for heart failure, acute myocardial infarction, or pneumonia. JAMA. Jan 23 2013;309(4):355-363.

6. Williams MV. A requirement to reduce readmissions: take care of the patient, not just the disease. JAMA. Jan 23 2013;309(4):394-396.

7. Fontanarosa PB, McNutt RA. Revisiting hospital readmissions. JAMA. 2013;309(4):398-400.

8. Ward BW, Schiller JS, Goodman RA. Multiple Chronic Conditions Among US Adults: A 2012 Update. Prev Chronic Dis. 2014;11:130389 (http://dx.doi.org/10.5888/pcd11.130389).

9. Gerteis J, Izrael D, Deitz D, et al. Multiple Chronic Conditions Chartbook. AHRQ Publications No, Q14-0038. Rockville, MD: Agency for Healthcare Research and Quality; April 2014. 
10. Alonso J, Ferrer M, Gandek B, et al. Health-related quality of life associated with chronic conditions in eight countries: results from the International Quality of Life Assessment (IQOLA) Project. Qual Life Res. Mar 2004;13(2):283-298.

11. Chapman DP, Perry GS, Strine TW. The vital link between chronic disease and depressive disorders. Preventing Chronic Disease. 2005;2(1):A14. Available from: http://www.cdc.gov/pcd/issues/2005/jan/04 0066.htm. Accessed April 1, 2015.

12. Carey TS, Crotty KA, Morrissey JP, et al. AHRQ Comparative Effectiveness Reviews. Future Research Needs for the Integration of Mental Health/Substance Abuse and Primary Care: Identification of Future Research Needs from Evidence Report/Technology Assessment No. 173. Rockville (MD): Agency for Healthcare Research and Quality (US); 2010.

13. Suffoletto B, Hu J, Guyette M, Callaway C. Factors contributing to emergency department care within 30 days of hospital discharge and potential ways to prevent it: differences in perspectives of patients, caregivers, and emergency physicians. JHM. May 2014;9(5):315-319. 14. Davis MM, Devoe M, Kansagara D, Nicolaidis C, Englander H. "Did I do as best as the system would let me?" Healthcare professional views on hospital to home care transitions. JGIM. Dec 2012;27(12):1649-1656.

15. Feigenbaum $P$, Neuwirth $E$, Trowbridge $L$, et al. Factors contributing to all-cause 30-day readmissions: a structured case series across 18 hospitals. Med Care. Jul 2012;50(7):599-605. 16. Jencks SF, Williams MV, Coleman EA. Rehospitalizations among Patients in the Medicare Fee-for-Service Program. NEJM. 2009;360(14):1418-1428. 
17. Arora VM, Prochaska ML, Farnan JM, et al. Problems after discharge and understanding of communication with their primary care physicians among hospitalized seniors: a mixed methods study. JHM. Sep 2010;5(7):385-391.

18. Coleman EA, Smith JD, Raha D, Min SJ. Posthospital medication discrepancies: prevalence and contributing factors. Arch Int Med. Sep 12 2005;165(16):1842-1847.

19. Foust JB, Naylor MD, Boling PA, Cappuzzo KA. Opportunities for improving post-hospital home medication management among older adults. Home Health Care Services Quarterly. 2005;24(1-2):101-122.

20. Coleman EA, Parry C, Chalmers S, Min SJ. The care transitions intervention: results of a randomized controlled trial. Arch Int Med. Sep 25 2006;166(17):1822-1828.

21. U.S. Department of Health \& Human Services: Centers for Medicare \& Medicaid Services. Readmissions Reduction Program. Page last Modified: 08/04/2014; Available from: https://www.cms.gov/medicare/medicare-fee-for-servicepayment/acuteinpatientpps/readmissions-reduction-program.html.

22. Naylor MD, Aiken LH, Kurtzman ET, Olds DM, Hirschman KB. The care span: The importance of transitional care in achieving health reform. Health Aff. Apr 2011;30(4):746-754.

23. Rennke S, Nguyen OK, Shoeb MH, Magan Y, Wachter RM, Ranji SR. Hospital-initiated transitional care interventions as a patient safety strategy: a systematic review. Ann Int Med. Mar 5 2013;158(5 Pt 2):433-440.

24. Feltner C, Jones CD, Cene CW, et al. AHRQ Comparative Effectiveness Reviews. Transitional Care Interventions To Prevent Readmissions for People With Heart Failure. Rockville (MD): Agency for Healthcare Research and Quality (US); 2014. 
25. Hickam DH, Weiss JW, Guise JM, et al. AHRQ Comparative Effectiveness Reviews.

Outpatient Case Management for Adults With Medical IIIness and Complex Care Needs.

Rockville (MD): Agency for Healthcare Research and Quality (US); 2013.

26. IOM (Insititute of Medicine). Initial National Priorities for Comparative Effectiveness

Research. Washington, D.C. June 30, 2009.

27. Bloink J, Adler KG. Transitional care management services: new codes, new requirements.

Fam Pract Manag. May-Jun 2013; 20(3):12-17.

28. U.S. Department of Health \& Human Services: Centers for Medicare \& Medicaid Services.

Medicare Learning Network: Transitional Care Management Services. June, 2013; Available at: http://www.nacns.org/docs/TransCareMgmtFAQ.pdf. Accessed April 1, 2015.

29. Coleman EA. The Care Transitions Program. 2007; Available from:

http://www.caretransitions.org/. Accessed April 1, 2015.

30. Hansen LO, Greenwald JL, Budnitz T, et al. Project BOOST: effectiveness of a multihospital effort to reduce rehospitalization. JHM. Aug 2013;8(8):421-427.

31. IOM (Insititute of Medicine). Finding What Works in Health Care: Standards for Systematic Reviews. Washington, D.C.: The National Academies Press, March 23, 2011.

32. Brown $\mathrm{P}$, Brunnhuber $\mathrm{K}$, Chalkidou $\mathrm{K}$, et al. How to formulate research recommendations. BMJ. 2006; 333(7572): 804-806.

33. Samson D, Schoelles KM. AHRQ Methods for Effective Health Care. Developing the Topic and Structuring Systematic Reviews of Medical Tests: Utility of PICOTS, Analytic Frameworks, Decision Trees, and Other Frameworks. In: Chang SM, Matchar DB, Smetana GW, Umscheid CA, 
eds. Methods Guide for Medical Test Reviews. Rockville (MD): Agency for Healthcare Research and Quality (US); 2012.

34. Tuso P, Huynh DN, Garofalo L, et al. The readmission reduction program of Kaiser Permanente Southern California-knowledge transfer and performance improvement. The Permanente Journal. Summer 2013;17(3):58-63.

35. White B, Carney PA, Flynn J, Marino M, Fields S. Reducing hospital readmissions through primary care practice transformation. JFP. Feb 2014;63(2):67-73.

36. Jackson CT, Trygstad TK, DeWalt DA, DuBard CA. Transitional care cut hospital readmissions for North Carolina Medicaid patients with complex chronic conditions. Health Aff. Aug 2013;32(8):1407-1415.

37. Hughes JS, Averill RF, Eisenhandler J, et al. Clinical Risk Groups (CRGs): a classification system for risk-adjusted capitation-based payment and health care management. Medical care. 2004;42(1):81-90.

38. van Walraven C, Dhalla IA, Bell C, et al. Derivation and validation of an index to predict early death or unplanned readmission after discharge from hospital to the community. CMAJ. 2010;182(6):551-557.

39. Ahmed OI, Rak DJ. Hospital readmission among participants in a transitional case management program. Am J Manag Care. Oct 2010;16(10):778-783.

40. Naylor MD, Brooten D, Campbell R, et al. Comprehensive discharge planning and home follow-up of hospitalized elders: a randomized clinical trial. JAMA. Feb1999;281(7):613-620. 41. Naylor MD, Brooten DA, Campbell RL, et al. Transitional Care of Older Adults Hospitalized with Heart Failure: A Randomized, Controlled Trial. J Am Geriatr Soc. 2004;52(5):675-684. 
42. Allen J, Hutchinson AM, Brown R, Livingston PM. Quality care outcomes following transitional care interventions for older people from hospital to home: a systematic review. BMC Health Services Research. 2014;14:346.

43. Burke RE, Guo R, Prochazka AV, Misky GJ. Identifying keys to success in reducing readmissions using the ideal transitions in care framework. BMC Health Services Research. 2014;14:423.

44. Laiteerapong N, Huang ES. The Pace of Change in Medical Practice and Health Policy: Collision or Coexistence? JGIM. 2015; 30(6):848-852.

45. Jacobson P, Jazowski S. Physicians, the Affordable Care Act, and Primary Care: Disruptive Change or Business as Usual? JGIM. 2011;26(8):934-937. 